\title{
Morbimortalidade Neonatal em Unidade de Terapia Intensiva
}

\author{
Neonatal Morbidity and Mortality in Intensive Care \\ Unit
}

\section{La morbilidad y mortandad neonatal en Unidad de Cuidados Intensivos}

Adrielle Dantas Santiago ${ }^{1}$ Maria Nice Dutra de Oliveira ${ }^{2}$

Lívia Lessa de Oliveira ${ }^{3}$

Elzo Pereira Pinto Junior ${ }^{4}$

RESUMO: Este estudo objetiva descrever as morbidades e mortalidade de recém-nascidos internados em Unidade de Terapia Intensiva Neonatal. Neste contexto, utilizou-se abordagem descritiva, transversal, usando dados dos prontuários dos recém-nascidos internados em Unidades de Terapia Intensiva Neonatal, com uma população constituída por trinta neonatos, e os dados foram analisados através de frequência simples e percentual. Os resultados identificaram que a maioria dos neonatos era do sexo masculino, com nascimento pré-termo e de parto vaginal. As afecções respiratórias foram as principais morbidades encontradas na unidade e o óbito foi o desfecho clínico de 23,1\% dos casos. As morbidades como a prematuridade pode trazer consequências respiratórias ao recém-nascido, e a unidade tem papel importante na assistência neonatal. Sugere-

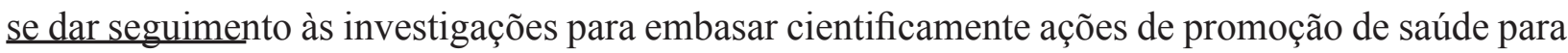
1 Physiotherapist. Universidade Estadual do Sudoeste da Bahia. Mailing address: E-mail:santiago.adrielle@hotmail. com

2 Assiatant Professor of the Universidade Estadual do Sudoeste da Bahia. E-mail: mnicedutra@gmail.com 3 Specialist in Pediatric and Neonatal Hospital Physiotherapy. Universidade Estadual do Sudoeste da Bahia. E-mail: livialessauesb@gmail.com

4 Master of Public Health. PhD in Public Health. Substitute Professor of the Public Health Institute - Universidade Federal da Bahia. E-mail: elzojr@hotmail.com 
as gestantes e ações de conscientização aos profissionais de saúde no município.

Palavras-chave: Recém-nascido. Epidemiologia. Unidades de Terapia Intensiva Neonatal.

\begin{abstract}
This study aims to describe the morbidity and mortality of neonates admitted to the Neonatal Intensive Care Unit. In this context, a descriptive, cross-sectional approach was used, using data from the charts of neonates hospitalized in a Neonatal Intensive Care Unit, with a population consisting of thirty neonates and data analyzed by simple and percentage frequency. The results identified that most of the neonates were males, with preterm birth and vaginal delivery. Respiratory diseases were the main morbidities found in the unit and the death was the clinical outcome of $23.1 \%$ of the cases. Morbidity such as prematurity can bring respiratory consequences to the newborn and the unit plays an important role in neonatal care. It is suggested to follow the investigations to scientifically base health promotion actions for pregnant women and awareness actions for health professionals in the municipality.
\end{abstract}

Keywords: newborn; epidemiology; neonatal intensive care units.

RESUMEN: Este estudio tiene como objetivo describir la morbilidad y mortandad de recién nacidos ingresados en la Unidad de Cuidados Intensivos Neonatales. En este contexto, se utilizó un enfoque descriptivo, transversal, usando los datos de las historias clínicas de recién nacidos ingresados en la Unidad de Cuidados Intensivos Neonatales, con una población de treinta neonatos. Se analizaron los datos a través de la secuencia simple y del porcentaje. Los resultados indicaron que la mayoría de los recién nacidos eran del sexo masculino, con el parto prematuro y el parto vaginal. Las enfermedades respiratorias fueron las principales morbilidades que se encuentran en la unidad y la muerte fue el resultado clínico en $23,1 \%$ de los casos. Las morbilidades como la prematuridad pueden traer consecuencias respiratorias para el recién nacido y la unidad juega un papel importante en la atención neonatal. Se sugiere dar seguimiento a las investigaciones científicas para apoyar las actividades de promoción de la salud a mujeres embarazadas y realizar campañas de concienciación a profesionales de la sanidad de la ciudad.

Palabras clave: recién nacido; epidemiología; unidades de cuidados intensivos neonatales.

\title{
INTRODUÇÃO
}

Os primeiros dias de vida de um recém-nascido são marcados por uma fase na qual há elevado risco de morbimortalidade, dada a sua condição de vulnerabilidade biológica. Nesse contexto, as Unidades de Terapia Intensiva Neonatais (UTIN) representam um ambiente de alta concentração de tecnologias de suporte à vida, prontas para fornecerem o cuidado nos mais diferenciados graus de complexidade e garantir o melhor manejo clínico dos recém-nascidos mais graves ${ }^{(1)}$. Nas UTIN, os distúrbios respiratórios em conjunto com infecções neonatais representam 50,8\% das morbidades apresentadas, já os distúrbios cardíacos correspondem a 8,4\% e 5,31\% dos bebês que nascem com algum tipo de malformação congênita ${ }^{(2,3)}$. Os principais motivos de internação dos recém-nascidos se dão por causas respiratórias, baixo peso ao nascimento, prematuridade e icterícia neonatal ${ }^{(2,3)}$. 
Essas morbidades podem ser reduzidas através das ações dos programas de saúde, como a Rede Cegonha e Método Canguru, por exemplo, os quais possibilitam uma assistência adequada do período gestacional até o nascer, e humanizam o atendimento com o intuito de melhorar a qualidade dos serviços de saúde ${ }^{(4)}$.

No Brasil, em 2015, a taxa de mortalidade neonatal foi de 8,9/1000 nascidos vivos. Essa taxa é relativamente alta quando comparada aos países desenvolvidos como Canadá (3,9/1000 nascidos vivos) e Estados Unidos (3,6/1000 nascidos vivos) $)^{(5)}$. Nos últimos anos, o Brasil vem reduzindo os índices de mortalidade infantil, no entanto, ainda se distancia bastante dos países mais desenvolvidos, tornando evidentes as lacunas existentes no que diz respeito à qualidade e ao alcance das ações do setor de saúde ${ }^{(6)}$. Lansky et al.(7) associaram os óbitos neonatais à prematuridade, ao baixo peso ao nascer, aos fatores de risco maternos, às malformações congênitas e à asfixia perinatal, os quais estão fortemente ligados à desqualificação do atendimento durante o pré-natal e o parto.

A presença de tecnologia de suporte à vida no cuidado intensivo neonatal, como fototerapia, ventilação mecânica e oxigenoterapia, aumentam as chances de sobrevida desses recém-nascidos de risco, o que evidencia a importância da UTIN nas maternidades ${ }^{(8)}$. Nesse contexto, tornase oportuno traçar o perfil de morbidade e mortalidade neonatal, pois a identificação de causas perinatais mais frequentes poderá dar subsídios para conscientizar e reforçar a assistência à gestante no período pré-natal e parto, evitando desfechos negativos para o neonato. Para isso, esse estudo objetivou descrever o perfil de morbimortalidade e intervenções nos recém-nascidos internados na UTIN, em um município do interior da Bahia.

\section{MATERIAL E MÉTODO}

Trata-se de um estudo epidemiológico, transversal e descritivo que teve como cenário a UTIN da Santa Casa Hospital São Judas Tadeu, situada no município de Jequié/BA, a qual presta assistência aos municípios que compõem essa microrregião. Inicialmente, existiam 42 neonatos internados na UTIN, entre março e junho de 2016, período referente à realização da pesquisa. Foram excluídos da pesquisa 12 neonatos cujos responsáveis não foram encontrados para a assinatura do Termo de Consentimento Livre e Esclarecido (TCLE) ou não aceitaram participar da pesquisa, totalizando uma amostra final de 30 recém-nascidos (RN).

A coleta de dados foi realizada através de preenchimento de um formulário desenvolvido pelos autores, com base nas variáveis que atendem aos objetivos da pesquisa, o qual recolheu informações do prontuário do recém-nascido, da Declaração de Nascido Vivo (DNV) e Declaração de Óbito (DO).

O formulário foi dividido em cinco blocos, os quais compreendem Dados Maternos, do Parto e Nascimento; Pós-parto imediato - Intercorrências e intervenções; Pós-parto tardio - Intercorrências e intervenções; Alta hospitalar e Óbito. 
Para a investigação das causas de internação, foi realizada a categorização das patologias com base na Classificação Internacional das Doenças - $10^{\mathrm{a}}$ revisão (CID - 10). Desse modo, ressalta-se que existe a possibilidade de um mesmo indivíduo da pesquisa apresentar mais de uma causa de internação. A variável "Peso ao nascer" foi classificada em Adequado $(\geq 2500 \mathrm{~g})$, Baixo peso (1500g a $2499 \mathrm{~g})$, Muito baixo peso (1000g a $1499 \mathrm{~g})$ e Extremo baixo peso $(<1000 \mathrm{~g})$. Na investigação, as intervenções utilizadas considerou-se terapia medicamentosa, o uso de sedativos, antibióticos, analgésicos, anticonvulsivantes, antirrefluxo, diuréticos e broncodilatadores. Os óbitos foram classificados como neonatal precoce ( 0 a 6 dias) e neonatal tardio ( 7 a 27 dias).

Os dados coletados foram analisados através do programa SPSS ${ }^{\circledR}$ versão 21.0. Na análise foi utilizada a frequência absoluta e relativa. O estudo foi aprovado pelo Comitê de Ética em Pesquisa da Universidade Estadual do Sudoeste da Bahia (CEP/UESB) sob CAAE 49106915.1.0000.0055. Os responsáveis legais assinaram o Termo de Consentimento Livre e Esclarecido, e todas as exigências da Resolução No 466 de dezembro de 2012, do Conselho Nacional de Saúde foram atendidas.

\section{RESULTADOS}

Dentre os 30 neonatos estudados, $80,0 \%$ pertenciam ao sexo masculino, $56,7 \%$ nasceram de parto pré-termo e 43,3\% obtiveram Índice de Apgar no $5^{\circ}$ minuto considerado normal (8 a 10$)$. No que se refere ao tipo de parto e nascimento, 70\% nasceram de parto vaginal e 40\% apresentaram peso adequado à idade gestacional. Em relação às características maternas, observou-se que 53,3\% das mães se encontravam na faixa etária de 20 a 35 anos e 53,3\% eram naturais do município de Jequié/BA (Tabela 1).

Tabela 1 - Características do recém-nascido, do parto e maternas, Jequié/BA, 2016

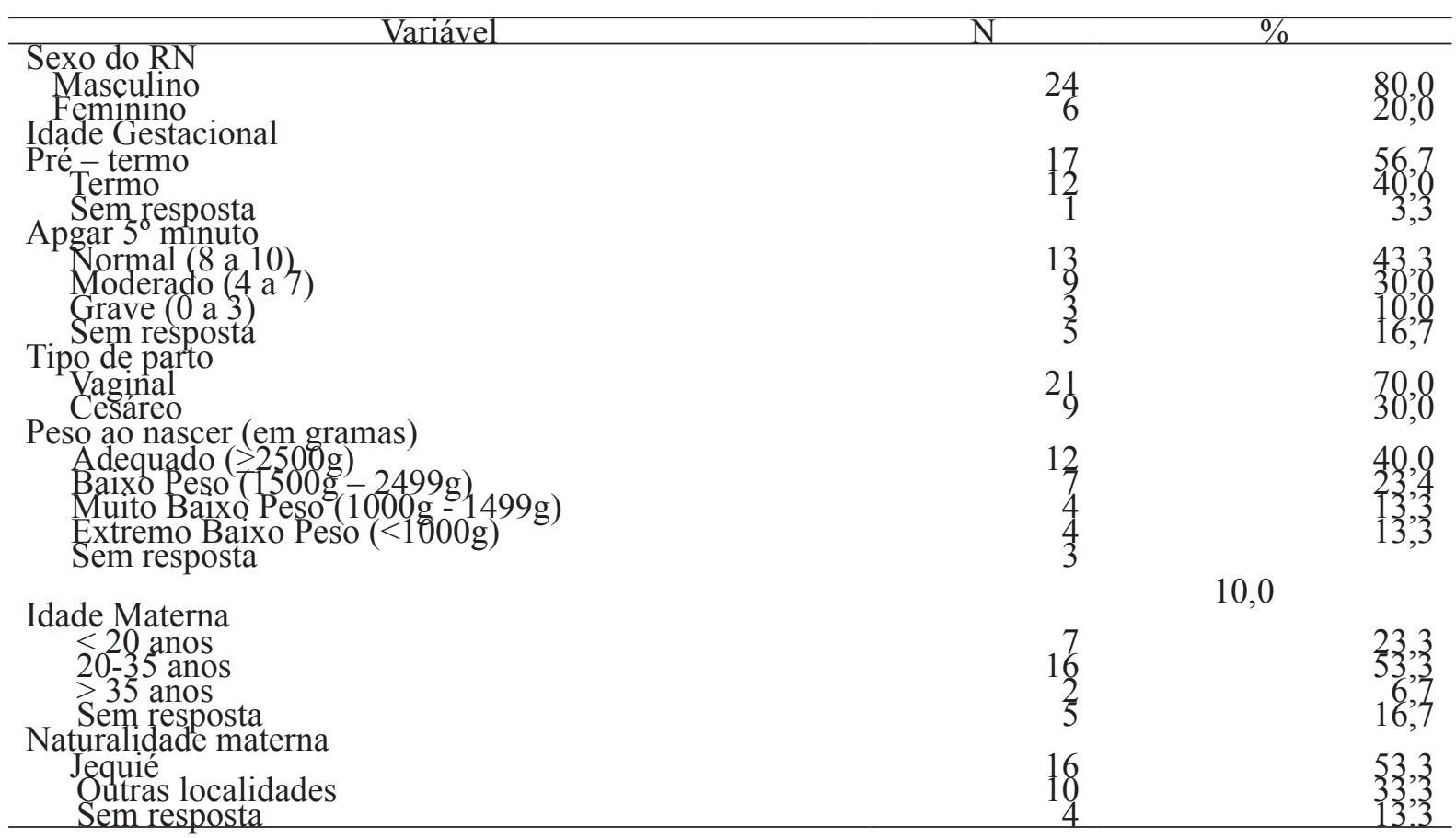


Quanto ao motivo de internação, que poderia ser mais de um motivo, a maioria dos neonatos deu entrada na unidade com algum tipo de afecção respiratória $(93,3 \%)$ ou baixo peso ao nascimento $(43,3 \%)$ (Figura 1).

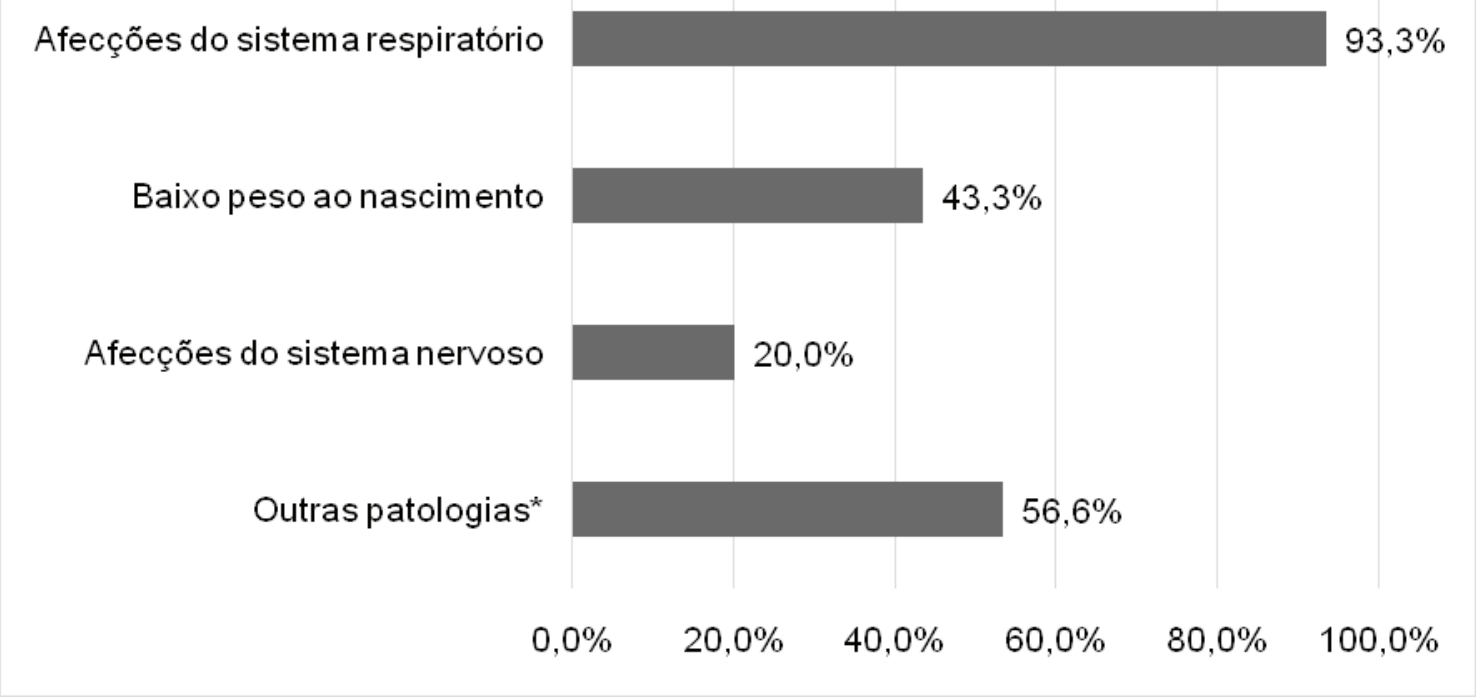

Figura 1 - Causas de internação em Unidade de Terapia Intensiva Neonatal, Jequié/BA, 2016

*Hemorragia digestiva, Choque Hipovolêmico, Sepse, Hipoglicemia e Icterícia

Verificou-se que as principais morbidades encontradas na unidade foram prematuridade (56,7\%), anóxia/hipóxia (53,3\%) e Doença da Membrana Hialina (DMH) (43,3\%), enquanto que as malformações congênitas $(10 \%)$, apnéia $(3,3 \%)$ e tocotrauma $(3,3 \%)$ ocorreram em menor frequência. (Figura 2)

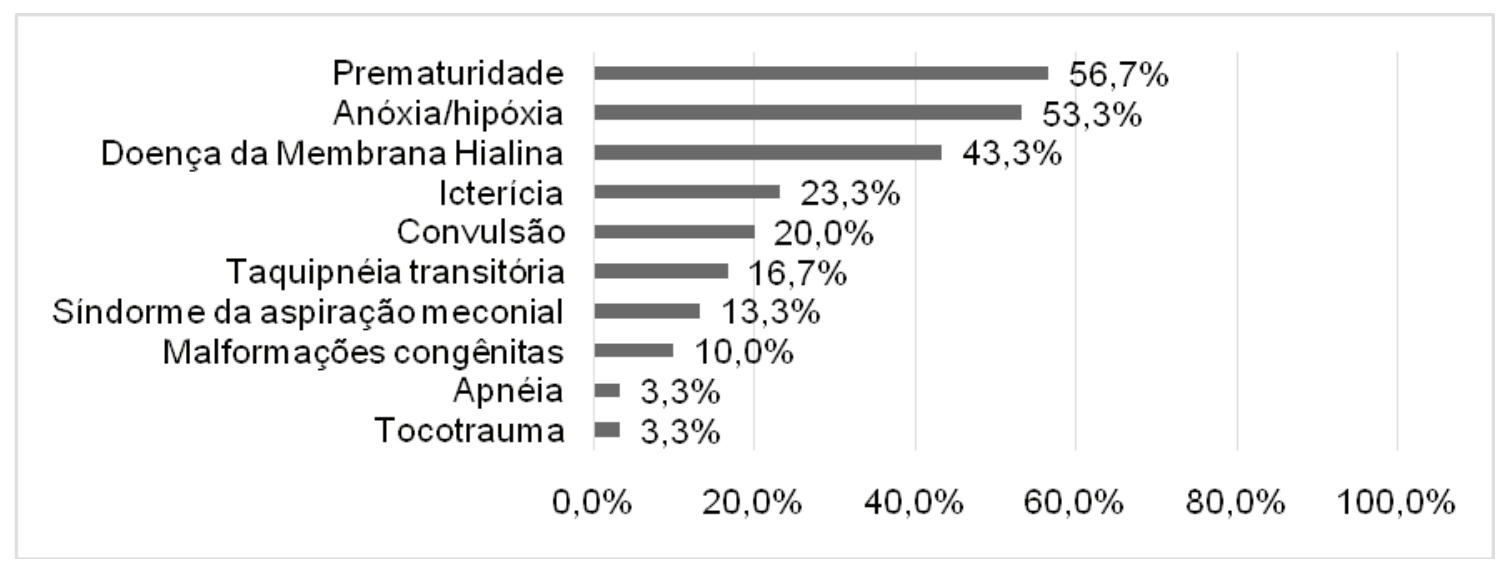

Figura 2 - Principais morbidades neonatais em Unidade de Terapia Intensiva, Jequié/BA, 2016.

Em decorrência das morbidades apresentadas nos neonatos, algumas intervenções foram realizadas, destacando-se o uso de medicações (93,3\%), a aspiração de vias aéreas superiores (73,3\%), oxigenoterapia (50\%) e Ventilação Mecânica Invasiva (50\%) (Figura 3). 


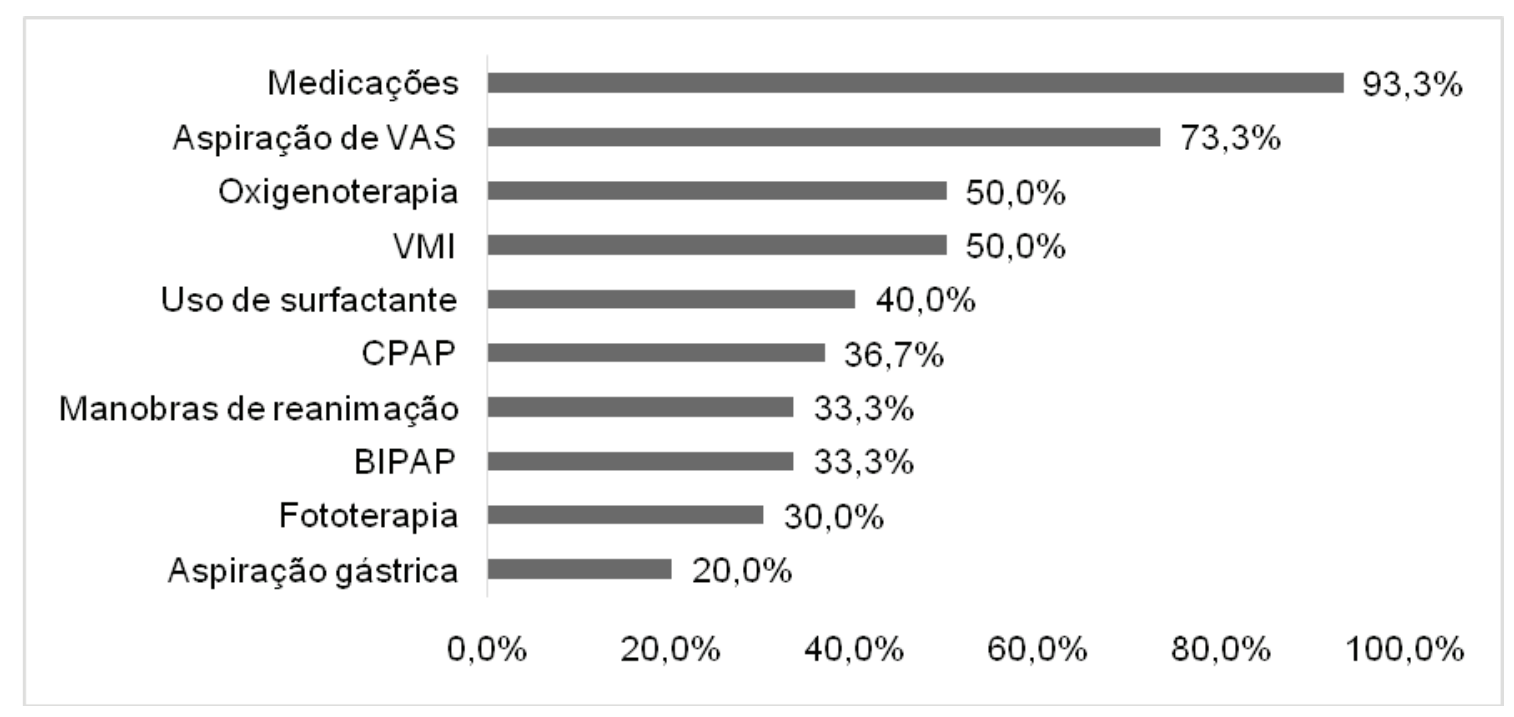

Figura 3 - Intervenções em neonatos em Unidade de Intensiva, Jequié/BA, 2016

VAS: Vias aéreas superiores; VMI: Ventilação mecânica invasiva; CPAP: Continuous positive airwaypressure; BIPAP: Bilevel Positive PressureAirway

Quanto ao desfecho clínico dos neonatos estudados, observou-se que 42,3\% (11) receberam alta da unidade antes de completar 28 dias de vida; 34,6\% (9) continuaram internados e 23,1\% (6) evoluíram para óbito (Figura 4).

Figura 4 - Desfecho clínico de recém-nascido após 28 dias da hospitalização em Unidade de Terapia Intensiva Neonatal ( $\mathrm{n}=26)$, Jequié/BA, 2016

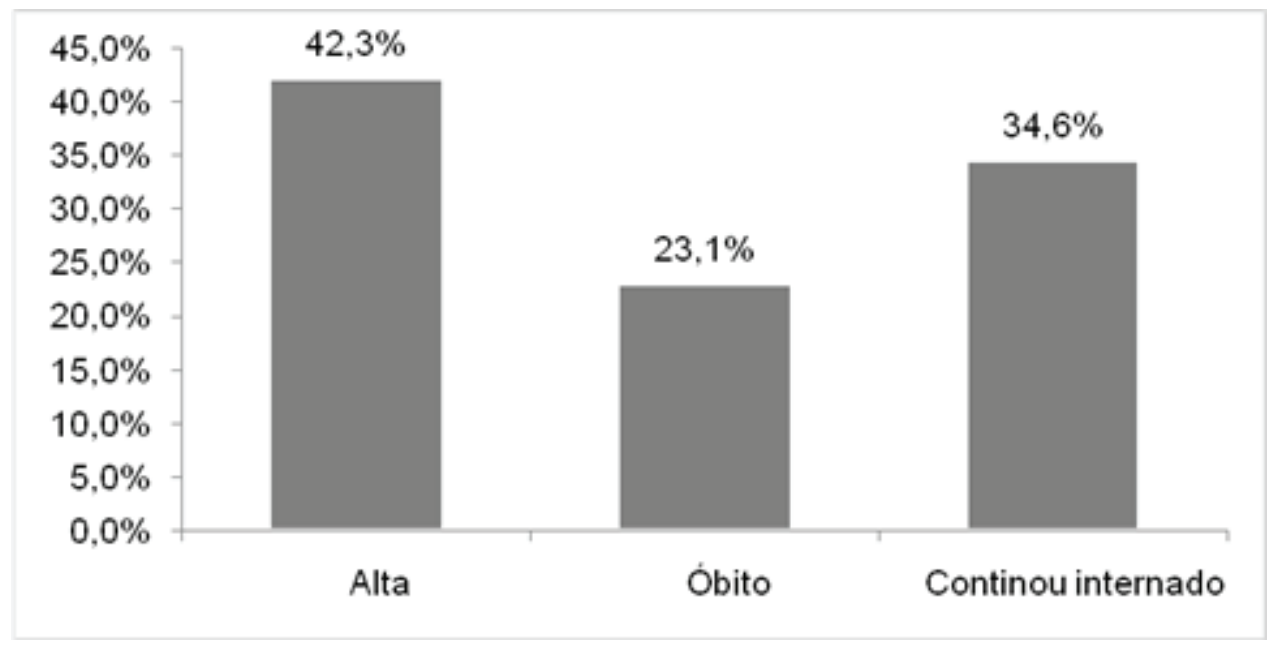

\section{DISCUSSÃO}

Os recém-nascidos (RN) admitidos em uma Unidade de Terapia Intensiva (UTIN) do município de Jequié/BA foram, em sua maioria, do sexo masculino, de nascimento pré-termo, 
através de parto vaginal, com Índice de Apgar considerado normal e peso ao nascer $\leq 1500 \mathrm{~g}$. As causas mais importantes de internação estiveram relacionadas às afecções respiratórias, enquanto a prematuridade foi a morbidade encontrada com maior frequência na unidade. A maioria dos neonatos recebeu alta clínica, apesar das morbidades apresentadas durante o período.

A predominância da população estudada foi do sexo masculino, assim como outros estudos realizados no norte, nordeste e sul do país ${ }^{(3,10,11)}$. No que diz respeito à idade gestacional, houve predomínio de nascimentos pré-termos, corroborando com os outros estudos da literatura, os quais apontaram $66 \%$ e $70 \%{ }^{(3,10)}$. Demais autores também evidenciaram a prematuridade em seus estudos, porém com uma prevalência maior, identificando $92,14 \%$ e $92,3 \%{ }^{(11,12)}$.

Em relação ao Índice de Apgar no $5^{\circ}$ minuto, identificou-se que a maioria dos neonatos obteve índice considerado normal (escore de 8 a 10), assim como os 86,16\% encontrados em um estudo no norte do país ${ }^{(10)}$ e os $98,1 \%$ de um estudo realizado nos Estados Unidos ${ }^{(13)}$. No presente estudo, havia prontuários com o Índice de Apgar não preenchido. Dessa maneira, é oportuno ressaltar a importância do preenchimento desse escore, uma vez que é preditor de mortalidade neonatal ${ }^{(14)}$.

Em relação ao tipo de parto, a maioria dos nascimentos ocorreu por via vaginal, confrontando com um estudo feito no norte do país, que apresentou o total de $44 \%$ de partos vaginais ${ }^{(10)}$. Outro estudo realizado em UTIN realizou análise bivariada do tipo de parto e óbito neonatal, concluindo que há maior risco de óbito em partos cesáreos ${ }^{(15)}$.

Há uma tendência no Brasil pelo parto cesáreo, como mostra um estudo nacional de base hospitalar, no qual, a cesariana representa $55,4 \%$ do total de partos ${ }^{(16)}$. Em decorrência disso, a Agência Nacional de Saúde (ANS) determinou obrigatório, durante o acompanhamento pré-natal, o esclarecimento à gestante sobre os riscos e benefícios dos tipos de parto $^{(17)}$. O número elevado de partos vaginais nessa unidade pode ser justificado pelo fato da maternidade possuir um Centro de Parto Normal (CPN) em conformidade com o programa Rede Cegonha.

Com relação ao peso ao nascimento, os neonatos apresentaram baixo peso, muito baixo peso ou extremo baixo peso. Outros estudos realizados no Brasil demonstraram um predomínio de neonatos internados em UTIN com peso ao nascer $\leq 1500 \mathrm{~g}^{(10,11)}$, diferentemente de países de primeiro mundo, onde $84,2 \%$ dos neonatos nascem com peso adequado ${ }^{(13)}$. Silva et al. ${ }^{(18)}$, com os dados da pesquisa Nascer no Brasil, evidenciaram que com peso ao nascer abaixo de $1.500 \mathrm{~g}$ apresentou forte associação com mortalidade neonatal.

Algumas morbidades como prematuridade e baixo peso ao nascer podem ser associadas com a idade materna, sendo essas mais comuns em recém-nascidos de mães com idade $\leq 20$ anos e $>$ $35 \operatorname{anos}^{(19)}$. Em um estudo de caso-controle realizado em outra UTIN no nordeste, foi observada predominância de idade materna entre 20 e 34 anos e ao associar a necessidade de internação do recém-nascido com a idade materna, não encontrou significância ${ }^{(20)}$. Outros estudos realizados em 
UTIN pelo país também encontraram maior proporção de mães com idade entre 20 e 35 anos ${ }^{(10,21)}$.

A maternidade onde foi realizado esse estudo é a única referência com UTIN para atender toda a microrregião, composta por 26 municípios. Apesar disso, foi observado que 53,3\% das mães são naturais de Jequié, onde está localizada a unidade. Nesse quesito, distancia-se do estudo realizado no estado do Ceará, o qual observou maior frequência de internações de neonatos cujas mães não residiam no mesmo local da $\operatorname{UTIN}^{(20)}$. Essa diferença pode ser explicada pelo fato de que, no estudo desenvolvido no Ceará, a unidade de referência se localizava na capital, de modo que esta serviria de referência para os casos mais graves de todo o Estado.

As causas de internações nesta unidade foram similares aos estudos em UTIN nos estados do Pará, Ceará e Rio Grande do Sul, nos quais as afecções respiratórias e baixo peso ao nascer estiveram entre os principais diagnósticos de admissão ${ }^{(3,10,11)}$. Nesses estudos também foram identificados a associação de mais de um fator causal de internação, o que evidencia a necessidade da atenção multiprofissional qualificada a esse RN de risco durante a internação e seguimento após alta.

A frequência das morbidades encontradas nessa pesquisa difere do estudo feito em uma unidade no Sul do Brasil, o qual observou um maior número de casos de infecções neonatais (sepse), seguido de distúrbios respiratórios e icterícia ${ }^{(3)}$. A prematuridade e as patologias relacionadas ao sistema respiratório apresentaram maior destaque nesse estudo e isso pode ser justificado pelo fato do recém-nascido pré-termo possuir um aparelho respiratório imaturo, tornando-o mais suscetível às patologias como Doença da Membrana Hialina, Taquipnéia Transitória e risco de infecções ${ }^{(22)}$. As intercorrências durante o parto também podem desencadear a Síndrome da Aspiração de Mecônio, a qual é comumente apresentada em $\mathrm{RN}$ a termo e pós-termo ${ }^{(22)}$.

Durante o parto, o neonato passa por uma transformação fisiológica do sistema cardiorrespiratório para adaptar-se ao novo ambiente de vida, portanto, eventuais morbidades perinatais podem dificultar esse processo, fazendo com que esse indivíduo necessite da tecnologia de suporte à vida oferecida na UTIN ${ }^{(8,23)}$. Apesar de o recém-nascido prematuro apresentar imaturidade de órgãos e sistemas, em longo prazo, o desenvolvimento neuropsicomotor dessas crianças pode não ser prejudicado, principalmente se estiverem num ambiente adequado para estimulação das suas capacidades $^{(24)}$.

As intervenções utilizadas com maior frequência para o controle das morbidades foram: uso de medicações, aspiração de vias aéreas superiores (VAS), oxigenoterapia e ventilação mecânica invasiva. Além dessas, há destaque também para a utilização da ventilação mecânica não invasiva (CPAP e BIPAP) e o uso de surfactante. A terapia medicamentosa foi realizada, principalmente, no período neonatal precoce, que se refere a um período importante para a estabilização clínica do recém-nascido.

O uso de oxigênio suplementar, de ventilação mecânica e de surfactante obteve valores parecidos 
com os encontrados em demais estudos no país ${ }^{(10,25)}$. A maior frequência de intervenções, que estão interligadas ao sistema respiratório, pode ser justificada pelo fato das afecções respiratórias terem sido a maior causa de internação na unidade.

No tocante à mortalidade infantil, o componente neonatal é o de maior magnitude, correspondendo a $70 \%$ das mortes $^{(23)}$. Nesse estudo, em relação ao desfecho clínico dos neonatos internados, 23,1\% evoluiu para óbito. Outros estudos realizados no norte e sudeste do Brasil encontraram percentual de mortalidade de $37,74 \%$ e $23,9 \%$, respectivamente ${ }^{(10,25)}$. O indicador mortalidade é um elemento importante para avaliar o desenvolvimento de uma região ou país e também subsidiar as políticas públicas, tanto em avaliação dos serviços quanto ao planejamento de saúde desta população em específico $^{(23)}$. Quanto às limitações encontradas nesse estudo, evidenciamos o período relativamente curto de realização da pesquisa e dificuldades encontradas com dados não preenchidos.

\section{CONCLUSÃO}

O presente estudo evidencia que as morbidades relacionadas ao sistema respiratório foram as principais causas de internações, e que o óbito foi um desfecho clínico importante, ressaltando a importância da implantação UTIN nesta região na assistência ao binômio materno infantil. Sugerese dar seguimento a essa investigação para embasar cientificamente ações de promoção de saúde para as gestantes, e reforço quanto à conscientização dos profissionais de saúde, ressaltando a assistência humanizada como um importante meio de qualificação do atendimento e redução da morbimortalidade neonatal.

\section{REFERÊNCIAS BIBLIOGRÁFICAS}

1 Silva LJ da, Silva LR da, Christoffel MM. Tecnologia e humanização na Unidade de Terapia Intensiva Neonatal: reflexões no contexto do processo saúde-doença. Rev da Esc Enferm da USP. 2009;43(3):684-9.

2 Basso CG, Neves ET, Silveira A. Associação entre realização de pré-natal e morbidade neonatal. Texto Context Enferm. 2012;21(2):269-76.

3 Tadielo BZ, Neves ET, Arrué AM, Silveira A da, Ribeiro AC, Tronco CS, et al. Morbidade e mortalidade de recém-nascidos em tratamento intensivo neonatal no sul do Brasil. Rev da Soc Bras Enfermeiros Pediatras [Internet]. 2013;13(1):7-12. Recuperado de: http://www.seer.ufsj.edu. br/index.php/recom/article/viewFile/401/566

4 Brasil. Ministério Da Saúde. Atenção ao pré-natal de baixo risco [Internet]. Editora do Ministério da Saúde, 2012. Ministério da Saúde; 2012. 318 p. Recuperado de: http://bvsms.saude. gov.br/bvs/publicacoes/cadernos_atencao_basica_32_prenatal.pdf

5 World Health of Organization. World Health Statistics: 2016. 2016. p. 103-20. 
6 Victora CG, Aquino EML, Leal M do C, Monteiro CA, Barros FC, Szwarcwald CL. Maternal and child health in Brazil: progress and challenges. Lancet [Internet]. Elsevier Ltd; 2011;377(9780):1863-76. Recuperado de: http://dx.doi.org/10.1016/S0140-6736(11)60138-4

7 Lansky S, Friche AA de L, Silva AAM da, Campos D, Bittencourt SD de A, Carvalho ML de, et al. Pesquisa nascer no Brasil : perfil da mortalidade neonatal e avaliação da assistência à gestante e ao recém-nascido. Cad Saúde Pública [Internet]. 2014;30(Sup):192-207. Recuperado de: http://www.scielo.br/scielo.php?pid=S0102311X2014001300024\&script=s ci_arttext

8 Gautam S, Agrawal A, Bangre A. Original article Impact of a tertiary sick newborn care unit on neonatal mortality and morbidity. Int J Healthc Biomed Res. 2016;4(3):55-9.

9 Organização Mundial da Saúde. CID-10 Classificação Estatística Internacional de Doenças e Problemas Relacionados à Saúde. $10^{\circ}$ ed. São Paulo: Universidade de São Paulo; 1997. 2 p.

10 Lima SS de, Silva SM da, Avila PES, Nicolau MV, Neves PFM das. Aspectos clínicos de recém-nascidos admitidos em Unidade de Terapia Intensiva de hospital de referência da Região Norte do Brasil. ABCS Heal Sci. 2015;40(3):62-8.

11 Magalhães FJ, Lima FET, Rolim KMC, Cardoso MVLML, Scherlock M do SM, Albuquerque NLS de. Respostas fisiológicas e comportamentais de recém-nascidos durante o manuseio em Unidade De Terapia Intensiva Neonatal. Rev Rene Fortaleza. 2011;12(1):136-43.

12 Granzotto JA, Mota DM, Real RF, Dias CM, Teixeira RF, Filho JCM, et al. Análise do perfil epidemiológico das internações em uma unidade de terapia intensiva neonatal. Revisa da Assoc Médica do Rio Gd do Sul. 2012;56(4):304-7.

13 Harrison W, Goodman D. Epidemiologic Trends in Neonatal Intensive Care, 2007-2012. JAMA Pediatr [Internet]. 2015;169(9):855-62. Recuperado de: http://archpedi.jamanetwork. com/article.aspx?articleid $=2381545$

14 Oliveira TG de, Freire PV, Moreira FT, Moraes J da SB de, Arrelaro RC, Rossi S, et al. Escore de Apgar e mortalidade neonatal em um hospital localizado na zona sul do município de São Paulo. Einstein. 2012;10(1):22-8.

15 Silva CF da, Leite ÁJM, Almeida NMGS de, Leon ACMP de, Olofin I, Catro ECM, et al. Fatores associados ao óbito neonatal de recém- nascidos de alto risco: estudo multicêntrico em Unidades Neonatais de Alto Risco no Nordeste brasileiro. Cad Saúde Pública. 2014;30(2):35568 . 
16 Domingues RMSM, Dias MAB, Nakamura-Pereira M, Torres JA, D’Orsi E, Pereira APE, et al. Processo de decisão pelo tipo de parto no Brasil : da preferência inicial das mulheres à via de parto final. Cad Saúde Pública. 2014;30:101-16.

17 Agência Nacional de Vigilância Sanitária (Brasil). Obrigatoriedade de os médicos entregarem a nota de orientação à gestante. nº 3982016.

18 Silva AAM da, Leite ÁJM, Lamy ZC, Moreira MEL, Gurgel RQ, Cunha AJLA da, et al. Morbidade neonatal near miss na pesquisa Nascer no Brasil. Cad Saude Publica [Internet]. 2014;30:182-91. Recuperado de: http://www.arca.fiocruz.br/handle/icict/9601

19 Gravena AAF, Paula MG de, Marcon SS, Carvalho MDB de, Pelloso SM. Idade materna e fatores associados a resultados perinatais. Acta Paul Enferm. 2013;26(2):130-5.

20 Costa AL do RR, Araujo Junior E, Lima JW de O, Costa F da S. Fatores de risco materno associados a necessidade de unidade de terapia intensiva neonatal. Rev Bras Ginecol e Obstet [Internet]. 2014;36(1):29-34. Recuperado de: http://www.scielo.br/scielo.php?script=sci_arttext\& pid $=$ S010072032014000100029

21 Bustamante T de F, Gonçalves T de A, Ferreira G, Moraes AG de. Estudo sobre a mortalidade em UTI Neonatal de um Hospital Escola no Sul de Minas. Rev Cência em Saúde [Internet]. 2014;4(2). Recuperado de: http://200.216.240.50:8484/rcsfmit/ojs-2.3.3-3/index.php/ rcsfmit_zero/article/view/231

22 Brasil. Ministério da Saúde. Atenção à Saúde do Recém-Nascido: guia para os profissionais de saúde. Ministério da Saúde; 2012. 195 p.

23 Brasil. Ministério da Saúde. Síntese de evidências para políticas de saúde: mortalidade perinatal. Ministério da Saúde; 2012. 44 p.

24 Ribeiro ASC, Formiga CKMR, David AC de. Healthy preterm infants: global motor coordination and early intervention. Fisioter em Mov [Internet]. 2015;28(1):85-95.

25 Carneiro JA, Vieira MM, Reis TC, Caldeira AP. Fatores de risco para a mortalidade de recém-nascidos de muito baixo peso em unidade de terapia intensiva neonatal. Rev Paul Pediatr. 2012;30(3):369-76.

Artigo apresentado em 24/01/2017

Artigo aprovado em 14/06/2017 Artigo publicado no sistema em 19/09/2017 